\title{
Asymmetric conjugate addition of ketones to nitroalkenes catalyzed by chiral bifunctional sulfamides
}

\author{
Sheng-ping Liu, Xue-jing Zhang, Jin-hua Lao and Ming Yan* \\ Industrial Institute of Fine Chemicals and Synthetic Drugs, School of Pharmaceutical Sciences, \\ Sun Yat-sen University, Guangzhou 510080, China \\ E-mail: yanming@mail.sysu.edu.cn
}

\begin{abstract}
Chiral bifunctional sulfamides were found to be efficient catalysts for the conjugate addition of ketones to nitroalkenes. Moderate to good enantioselectivities and yields were achieved for the reaction of acetone with a variety of $\beta$-aryl-nitroethylenes. Base additives were found to be more efficient than acid additives for the transformation. The reaction of methoxyacetone and cyclopentanone also provided the adducts with moderate enantioselectivities, however in low chemical yields.
\end{abstract}

Keywords: Chiral sulfamide, organocatalysis, nitroalkenes, ketone, conjugate addition

\section{Introduction}

In recent years asymmetric organocatalysis has emerged as a powerful tool for the synthesis of optically active compounds. ${ }^{1}$ A large number of asymmetric reaction have been achieved using chiral amines, thioureas (ureas) and other organic small molecules as the catalysts. Chiral bifunctional organocatalysts, which are capable of simultaneously activation of both the electrophiles and the nucleophiles, have proved to be superior for many asymmetric transformations. ${ }^{2-3}$ Chiral bifunctional thiourea-amine catalysts, developed by Jacobsen ${ }^{4}$ and the others $^{5-6}$, were found to be highly efficient for the conjugate addition of aldehydes and ketones to nitroalkenes. Excellent enantioselectivities and yields were achieved and the reaction provided an efficient strategy to prepare chiral $\gamma$-nitro ketones. ${ }^{7-8}$ However the preparation of these thiourea-amine catalysts generally requires tedious synthetic procedures, and the catalytic activity remains to be improved. Recently we developed a novel kind of chiral bifunctional sulfamide organocatalysts. The catalysts were readily prepared from amines and chiral cyclohexane-1,2-diamine with catechol sulfate. The stronger acidity of N-H bond of the sulfamide than the corresponding thiourea is expected to improve the catalytic activity. Excellent 
enantioselectivities and yields were obtained for the conjugate addition of aldehydes to nitroalkenes. ${ }^{9}$ As an extended study of the chiral sulfamide catalysts, herein we report their application in the asymmetric conjugate addition of ketones to nitroalkenes.

\section{Results and Discussion}

Chiral sulfamides 1a-1f were prepared according to previously reported procedures. ${ }^{9}$ The conjugate addition of acetone to trans- $\beta$-nitrostyrene $2 \mathbf{a}$ was carried out in the presence of 1a-1f and the results are summarized in Table 1. Although 1a was found to be highly efficient for the asymmetric conjugate addition of aldehydes to nitroalkenes, ${ }^{9}$ it provided the adduct product $4 \mathbf{a}$ in low yield and with moderate enantioselectivity (Table 1, entry 1). The yield was improved significantly by the addition of $20 \mathrm{~mol} \%$ benzoic acid (Table 1 , entry 2 ). It is noted that in the 1a-catalyzed addition of aldehydes to nitroalkenes, acid additives were detrimental and only base additives were efficient. ${ }^{9}$ The result implicated some inherent difference between the reaction of ketones and aldehydes. Sulfamides $\mathbf{1 b}, \mathbf{1 c}$ and $\mathbf{1 d}$ were also efficient for the reaction (Table 1, entries 3-5). Sulfamide 1b, derived from (S)-1-phenylethylamine and (R,R)-cyclohexane1,2-diamine, provided slightly better enantioselectivity and yield than 1a. In comparison, sulfamide 1c derived from (R)-1-phenylethylamine and $(R, R)$-cyclohexane-1,2-diamine, provided the adduct $\mathbf{4 a}$ in the same absolute configuration and with slightly lower enantioselectivity. The fact suggests that the enantioselective induction is mainly controlled by the chiral cyclohexanediamine unit. The additional chiral center only exerts small effects on the enantioselectivity. Sterically demanding $1 \mathbf{d}$ provided $\mathbf{4 a}$ with lower yield and enantioselectivity. Sulfamide 1e and 1f were totally inefficient for the reaction (Table 1, entries 6-7). The result confirms the importance of the primary amino group for the catalytic activity.<smiles>N[C@@H]1CCCC[C@H]1NS(=O)(=O)NCc1ccccc1</smiles>

$1 a$<smiles>N[C@@H]1CCCC[C@H]1NS(=O)(=O)NCc1cccc2ccccc12</smiles>

1d<smiles>C[C@@H](NS(=O)(=O)N[C@H]1CCCC[C@H]1N)c1ccccc1</smiles>

$1 b$<smiles>CC(=O)N[C@@H]1CCCC[C@@H]1NS(=O)(=O)NCc1ccccc1</smiles>

$1 e$<smiles>CC(NS(=O)(=O)N[C@H]1CCCC[C@H]1N)c1ccccc1</smiles>

1c

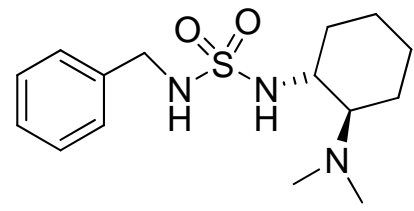

$1 f$

Scheme 1 
Table 1. The screening of chiral sulfamides 1a-1f ${ }^{\text {a }}$

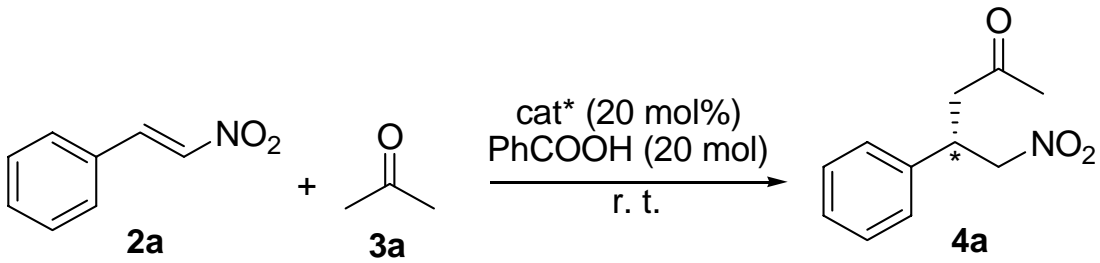

\begin{tabular}{ccccc}
\hline Entry & Catalyst & Time $(\mathrm{h})$ & Yield $(\%)^{\mathrm{b}}$ & Ee (\%), \\
\hline $1^{\mathrm{e}}$ & 1a & 79 & 37 & 64 \\
2 & 1a & 60 & 81 & 64 \\
3 & 1b & 60 & 84 & 66 \\
4 & 1c & 60 & 82 & 61 \\
5 & 1d & 60 & 61 & 51 \\
6 & 1e & 72 & n.r. ${ }^{\mathrm{f}}$ & - \\
7 & 1f & 72 & n.r. & - \\
\hline
\end{tabular}

${ }^{\mathrm{a}}$ The reactions were carried out with $2 \mathbf{2}(0.15 \mathrm{mmol}), \mathbf{1 a}-\mathbf{1 f}(0.03 \mathrm{mmol})$ in acetone $(0.3 \mathrm{~mL})$.

${ }^{\mathrm{b}}$ Isolated yields.

${ }^{\mathrm{c}}$ Determined by chiral HPLC analysis.

${ }^{\mathrm{d}}$ The absolute configuration of $\mathbf{4 a}$ was assigned to be $S$ by comparing the optical rotation with the reported data.

${ }^{\mathrm{e}}$ The reaction was carried out without benzoic acid.

${ }^{\mathrm{f}}$ No reaction.

A number of solvents were then screened using $\mathbf{1 b}$ as the catalyst and the results are listed in Table 2. The reaction solvent exerted significant effect on the yield and enantioselectivity. Generally the polar solvents resulted in lower yields and enantioselectivities (Table 2, entries 1-4). Water could also be used as the reaction solvent and provided the product with good enantioselectivity (71\% ee), however in low yield (Table 2, entry 2 ). The best yield (87\%) was achieved in ether (Table 2, entry 6), however the best enantioselectivity ( $84 \%$ ee) was obtained in toluene (Table 2, entry 9). Thus $\mathrm{Et}_{2} \mathrm{O}$ and toluene were selected for further optimization study. 
Table 2. The effect of reaction solvents ${ }^{\mathrm{a}}$

\begin{tabular}{cccc}
\hline Entry & Solvent & Yield (\%) & Ee (\%) \\
\hline 1 & $\mathrm{DMF}$ & 74 & 24 \\
2 & $\mathrm{H} 2 \mathrm{O}$ & 42 & 71 \\
3 & $\mathrm{MeOH}$ & 68 & 55 \\
4 & $\mathrm{CH}_{3} \mathrm{CN}$ & 39 & 50 \\
5 & $\mathrm{THF}$ & 84 & 54 \\
6 & $\mathrm{Et}{ }_{2} \mathrm{O}$ & 87 & 74 \\
7 & $\mathrm{CHCl}_{3}$ & 64 & 46 \\
8 & $\mathrm{CH}_{2} \mathrm{Cl}_{2}$ & 74 & 74 \\
9 & toluene & 55 & 84 \\
\hline
\end{tabular}

${ }^{\mathrm{a}}$ The reactions were carried out at room temperature with $\mathbf{2 a}(0.15 \mathrm{mmol}), \mathbf{1 b}(0.03 \mathrm{mmol})$, $\mathrm{PhCOOH}(0.03 \mathrm{mmol})$, acetone $(0.2 \mathrm{~mL})$ in solvent $(0.4 \mathrm{~mL})$.

In the organocatalytic conjugate addition of ketones to nitroalkenes, Brønsted acids were generally used as the efficient additives. ${ }^{10}$ However in our recent study of asymmetric conjugate addition of aldehydes to nitroalkenes catalyzed by chiral sulfamides, bases such as DIPEA and DMAP, were found to be extremely efficient additives. ${ }^{9}$ To obtain a clear understanding of the additive effect, a range of acid and base additives were examined in the reaction of $2 \mathbf{a}$ with acetone. The results are summarized in Table 3. Initially benzoic acid, acetic acid, $p$-nitro-benzoic acid, trifluoroacetic acid and (d)-camphor-10-sulfonic acid were studied in toluene (Table 3, entries 1-5). p-Nitro-benzoic acid provided the product 4a with good enantioselectivity, but the yield was poor. Trifluoroacetic acid and (d)-camphor-10-sulfonic acid were inefficient and no product could be obtained. Base additives such as imidazole, DIPEA, TEA and DMAP, were also examined (Table 3, entries 6-9). Imidazole provided 4a with good yield and enantioselectivity. DMAP afforded better enantioselectivity, however in lower yield. DIPEA and TEA provided 4a in low yields (Table 3, entries 8 and 9). Furthermore the acid and base additives were examined in ether and similar results were observed (Table 3, entries 10-18). Finally imidazole/ether system was selected for further study (Table 3, entry 15). 
Table 3. The effect of additives ${ }^{a}$

\begin{tabular}{ccccc}
\hline Entry & Solvent & Additive & Yield (\%) & Ee (\%) \\
\hline 1 & toluene & PhCOOH & 55 & 84 \\
2 & toluene & $\mathrm{AcOH}$ & 13 & 74 \\
3 & toluene & $p-\mathrm{NO}_{2} \mathrm{C}_{6} \mathrm{H}_{4} \mathrm{COOH}$ & 18 & 86 \\
4 & toluene & $\mathrm{CF}_{3} \mathrm{COOH}$ & - & - \\
5 & toluene & Camphor-10-sulfonic acid & - & - \\
6 & toluene & Imidazole & 80 & 78 \\
7 & toluene & DMAP & 58 & 86 \\
8 & toluene & DIPEA & 36 & 77 \\
9 & toluene & Et & 13 & 76 \\
10 & $\mathrm{Et}_{2} \mathrm{O}$ & $\mathrm{PhCOOH}_{13}$ & 87 & 74 \\
11 & $\mathrm{Et}_{2} \mathrm{O}$ & $\mathrm{AcOH}$ & 32 & 62 \\
12 & $\mathrm{Et}_{2} \mathrm{O}$ & $p-\mathrm{NO}_{2} \mathrm{C}_{6} \mathrm{H}_{4} \mathrm{COOH}$ & 47 & 70 \\
13 & $\mathrm{Et}_{2} \mathrm{O}$ & $\mathrm{CF}_{3} \mathrm{COOH}$ & 10 & 73 \\
14 & $\mathrm{Et}_{2} \mathrm{O}$ & $\mathrm{Camphor}-10$-sulfonic acid & 5 & 70 \\
15 & $\mathrm{Et}_{2} \mathrm{O}$ & $\mathrm{Imidazole}^{2}$ & 73 & 84 \\
16 & $\mathrm{Et}_{2} \mathrm{O}$ & $\mathrm{DMAP}^{2}$ & 32 & 67 \\
17 & $\mathrm{Et}_{2} \mathrm{O}$ & $\mathrm{DIPEA}_{13}$ & 6 & 64 \\
18 & $\mathrm{Et}_{2} \mathrm{O}$ & Et & - & - \\
\hline
\end{tabular}

${ }^{\mathrm{a}}$ The reactions were carried out at room temperature with $\mathbf{2 a}(0.15 \mathrm{mmol}), \mathbf{1 b}(0.03 \mathrm{mmol})$, additive $(0.03 \mathrm{mmol})$, acetone $(0.2 \mathrm{~mL})$ in solvent $(0.4 \mathrm{~mL})$.

The scope of the reaction was studied with a variety of $\beta$-aryl-nitroethylenes and ketones and the results are summarized in Table 4 . The reaction of substituted $\beta$-phenyl-nitroethylenes with acetone provided the adducts with moderate yields and enantioselectivities (table 4, entries 2-7). ortho, meta and para-chlorophenyl-nitroethylene afforded the product with similar enantioselectivity and yield (Table 4, entries 2-4). 2-Nitrophenyl-nitroethylene 2e gave the product with moderate enantioselectivity and in low yield. 4-Methoxylphenyl-nitroethylene $2 \mathrm{f}$ and 4-methylphenyl-nitroethylene $\mathbf{2 g}$ provided the products in the same yield, but $\mathbf{2} \mathbf{f}$ afforded better enantioselectivity. Heteroaryl-nitroethylenes, such as $\mathbf{2 h}$ and $\mathbf{2} \mathbf{i}$, were also applicable in the transformation. The corresponding adducts were obtained with good yields and moderate enantioselectivities (Table 4, entry 8-9). While methoxyacetone was used in the reaction with 2a, two diastereoisomeric adducts were obtained with moderate enantioselectivity and yield (Table 4, entry 10). The reaction of cyclopentanone gave the adduct with good enantioselectivity, however in low yield (Table 4, entry 11). 
Table 4. The reaction of $\beta$-aryl-nitroethylenes and ketones catalyzed by $\mathbf{1 b}^{\mathrm{a}}$

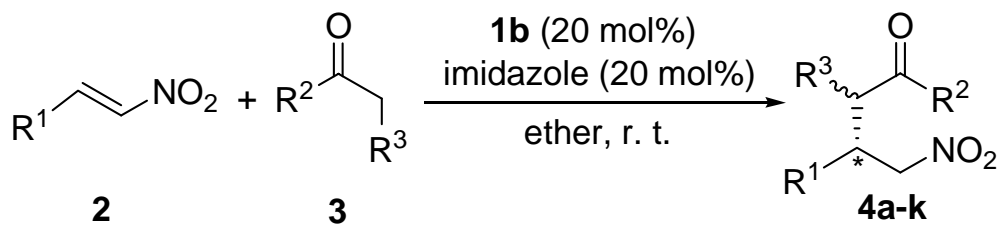

\begin{tabular}{ccccccc}
\hline Entry & $\mathrm{R}^{1}$ & $\mathrm{R}^{2}$ & $\mathrm{R}^{3}$ & Time $(\mathrm{h})$ & Product $($ yield \%) & Ee (\%) \\
\hline 1 & $\mathrm{Ph}, \mathbf{2 a}$ & $\mathrm{CH}_{3}$ & $\mathrm{H}$ & 60 & $\mathbf{4 a}(73)$ & 84 \\
2 & 2-Cl-Ph, 2b & $\mathrm{CH}_{3}$ & $\mathrm{H}$ & 49 & $\mathbf{4 b}(56)$ & 73 \\
3 & 3-Cl-Ph, 2c & $\mathrm{CH}_{3}$ & $\mathrm{H}$ & 94 & $\mathbf{4 c}(66)$ & 66 \\
4 & 4-Cl-Ph, 2d & $\mathrm{CH}_{3}$ & $\mathrm{H}$ & 60 & $\mathbf{4 d}(60)$ & 70 \\
5 & 2-NO2-Ph, 2e & $\mathrm{CH}_{3}$ & $\mathrm{H}$ & 70 & $\mathbf{4 e ~ ( 3 8 )}$ & 75 \\
6 & 4-OMe-Ph, 2f & $\mathrm{CH}_{3}$ & $\mathrm{H}$ & 60 & $\mathbf{4 f}(58)$ & 84 \\
7 & 4-Me-Ph, 2g & $\mathrm{CH}_{3}$ & $\mathrm{H}$ & 60 & $\mathbf{4 g}(58)$ & 68 \\
8 & 2-furanyl, 2h & $\mathrm{CH}_{3}$ & $\mathrm{H}$ & 60 & $\mathbf{4 h}(72)$ & 75 \\
9 & 2-thiophenyl, 2i & $\mathrm{CH}_{3}$ & $\mathrm{H}$ & 60 & $\mathbf{4 i}(70)$ & 69 \\
10 & Ph, 2a & $\mathrm{CH}_{3}$ & $\mathrm{OMe}$ & 60 & $\mathbf{4 j}(58)$ & $75 / 26^{\mathrm{d}}$ \\
11 & Ph, 2a & $-\mathrm{CH}_{2} \mathrm{CH}_{2} \mathrm{CH}{ }^{-}$ & 94 & $\mathbf{4 k}(35)$ & $84 / 74^{\mathrm{e}}$ \\
\hline
\end{tabular}

${ }^{\mathrm{a}}$ The reactions were carried out at room temperature with $2(0.15 \mathrm{mmol}), 3(0.2 \mathrm{~mL}), \mathbf{1 b}(0.03$ mmol $)$, imidazole $(0.03 \mathrm{mmol})$ in ether $(0.4 \mathrm{~mL})$.

${ }^{\mathrm{b}}$ Isolated yields.

${ }^{\mathrm{c}}$ Determined by chiral HPLC analysis.

${ }^{\mathrm{d}}$ Syn:anti $=43: 57$ as determined by ${ }^{1} \mathrm{H}$ NMR analysis.

${ }^{\mathrm{e}}$ Syn:anti $=43: 57$ as determined by ${ }^{1} \mathrm{H}$ NMR analysis.

Several other ketones, including cyclohexanone, acetophenone, 2-butanone, were examined in the reaction with $\beta$-nitrostyrene under the optimized reaction conditions, however the reactions were sluggish and almost no product was obtained. Using benzoic acid as the additive did not provide any improvements. The reactions of $\beta$-cyclohexyl nitroethylene and $\beta$-butyl 
nitroethylene with acetone were also studied, but only trace of the products was obtained.

A catalytic mechanism of chiral sulfamides is proposed in Scheme $2 .{ }^{5 \mathrm{c}}$ An imine intermediate $\mathbf{A}$ is generated from the catalyst $\mathbf{1 b}$ and acetone. The tautomerization of $\mathbf{A}$ is promoted by the base additive and provides the enamine B. Hydrogen bonding between the nitro group of nitrostyrene with the sulfamide (intermediate $\mathbf{C}$ ), increases the nucleophilicity of nitrostyrene. The neucleophilic attack of enamine occurs from si face of double bond and provides intermediate $\mathbf{D}$. The consequent proton transfer and hydrolysis give the product and regenerate the catalyst $\mathbf{1 b}$. The base additive is proposed to accelerate also the proton transfer step by removing proton from the imine cation.

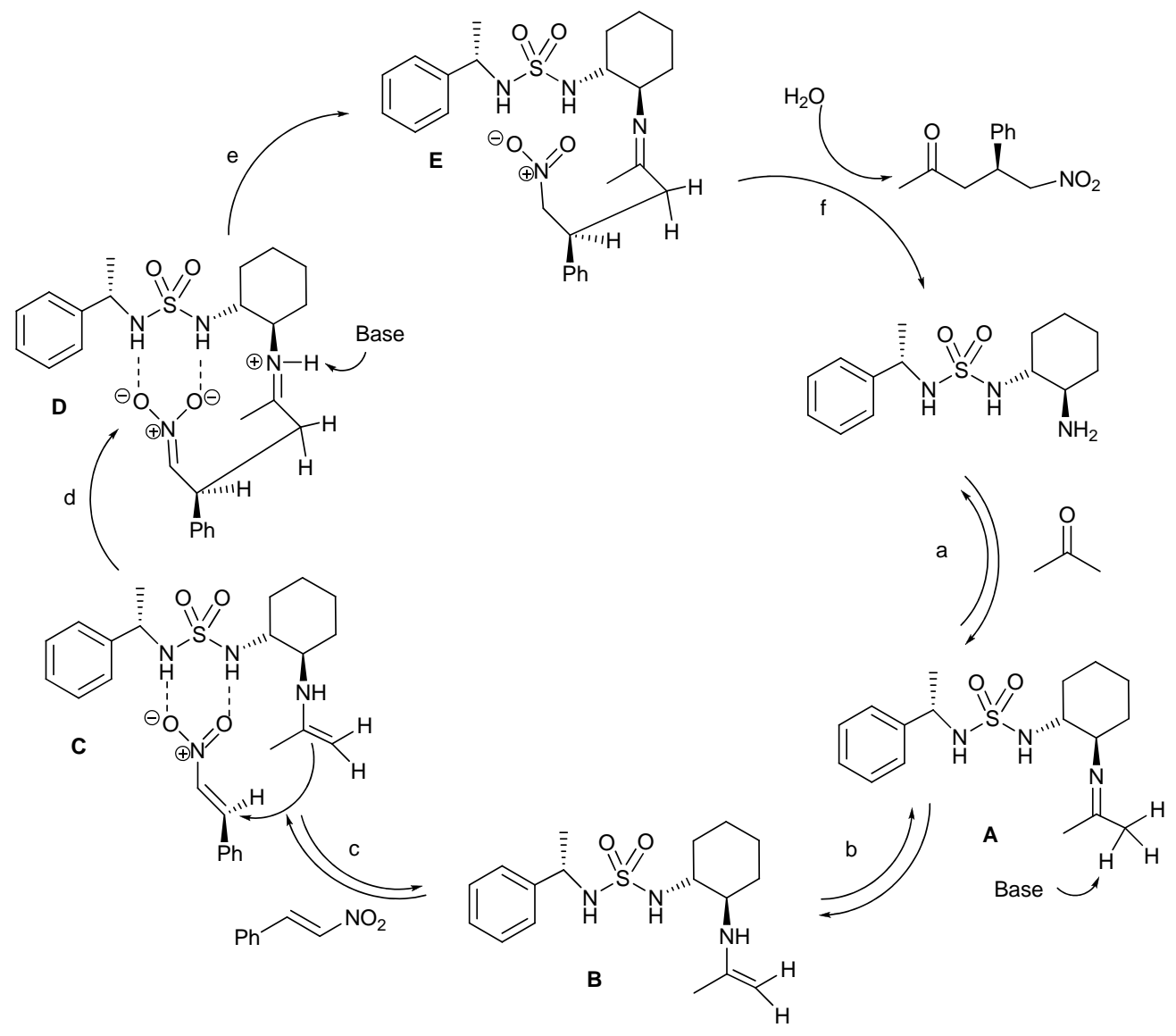

\section{Scheme 2}

\section{Conclusions}

In summary, chiral bifunctional sulfamides were found to be efficient catalysts for the conjugate addition of ketones to nitroalkenes. Moderate to good enantioselectivities and yields were achieved for the reaction of acetone with a variety of $\beta$-aryl-nitroethylenes. Methoxyacetone and 
cyclopentanone were also applicable in the transformation with lower chemical yields. Base additives were found to be more efficient than acid additives for the reaction. Further development of more efficient chiral sulfamide catalysts is currently under investigation.

\section{Experimental Section}

General Procedures. All solvents were used as commercial anhydrous grade without further purification. All reactions were carried out under open air. ${ }^{1} \mathrm{H}$ NMR spectra and ${ }^{13} \mathrm{C}$ NMR spectra were recorded on a Bruker AVANCE $400 \mathrm{MHz}$ spectrometer. High-resolution mass spectra were obtained with the Thermo MAT 95XP mass spectrometer. The low resolution mass spectra were obtained at the Thermo Trace GC Ultra-DSQ. Infrared (IR) spectra were recorded on a Bruker Tensor 37 spectrophotometer. Enantiomeric excesses were determined by HPLC using Daicel Chiralcel AS-H and OD-H column and eluting with ${ }^{n-}$ hexane $/{ }^{i-} \mathrm{PrOH}$ solution.

\section{Typical procedure for the asymmetric conjugate addition}

A solution of nitroalkene $(0.15 \mathrm{mmol}), \mathbf{1 b}(0.03 \mathrm{mmol})$, imidazole $(0.03 \mathrm{mmol})$ and ketone $(0.2$ $\mathrm{mL})$ in ether $(0.4 \mathrm{~mL})$ was stirred at room temperature for $60 \mathrm{~h}$. The reaction solution was concentrated under vacuum. The residue was purified by flash column chromatography over silica gel (petroleum ether/ethyl acetate $=6 / 1$ ) to give the product.

(S)-5-Nitro-4-phenylpentan-2-one (4a). ${ }^{4 a}$ White solid (73\%), m.p. $114-116{ }^{\circ} \mathrm{C}$ (lit ${ }^{4 \mathrm{a}}$. m.p. 120 $\left.-122.5{ }^{\circ} \mathrm{C}\right),[\alpha]_{\mathrm{D}}{ }^{20}=+1.7\left(\mathrm{c} 1.0, \mathrm{CHCl}_{3}\right)\left(\mathrm{lit}^{4 \mathrm{a}} \cdot[\alpha]_{\mathrm{D}}{ }^{20}=+2.4\right.$, c $1.03, \mathrm{CHCl}_{3}, 99 \%$ ee $) .{ }^{1} \mathrm{H} \mathrm{NMR}$ $\left(400 \mathrm{MHz}, \mathrm{CDCl}_{3}\right): \delta$ 7.35-7.20 $(5 \mathrm{H}, \mathrm{m}, \mathrm{Ar}-\mathrm{H}), 4.70\left(1 \mathrm{H}, \mathrm{dd}, \mathrm{J}=12.2,6.8 \mathrm{~Hz}, \mathrm{CH}_{2} \mathrm{NO}_{2}\right), 4.60$ $\left(1 \mathrm{H}, \mathrm{dd}, \mathrm{J}=12.2,7.8 \mathrm{~Hz}, \mathrm{CH}_{2} \mathrm{NO}_{2}\right), 4.01(1 \mathrm{H}, \mathrm{m}, \mathrm{CHAr}), 2.92\left(2 \mathrm{H}, \mathrm{d}, \mathrm{J}=7.0 \mathrm{~Hz}, \mathrm{CH}_{2}\right), 2.13$ $\left(3 \mathrm{H}, \mathrm{s}, \mathrm{CH}_{3}\right) ;{ }^{13} \mathrm{C}$ NMR $\left(100 \mathrm{MHz}, \mathrm{CDCl}_{3}\right): \delta 205.4(\mathrm{C}=\mathrm{O}), 138.8\left(\mathrm{C}_{\text {quat }}\right), 129.1(2 \mathrm{CH}), 127.9$ $(\mathrm{CH}), 127.4(2 \mathrm{CH}), 79.5\left(\mathrm{CH}_{2}\right), 46.1(\mathrm{CH}), 39.0\left(\mathrm{CH}_{2}\right), 30.4\left(\mathrm{CH}_{3}\right)$; IR $(\mathrm{KBr}):$ 3040, 2950, 2920, 1715, 1546, 1384, 1362, 1162, 758, $696 \mathrm{~cm}^{-1}$; MS (EI): $207.0\left(\mathrm{M}^{+}\right), 190.8,166.9,132.9,91.0$, 84.0. The enantiomeric excess was determined by HPLC with an AS-H column $\left({ }^{n-}\right.$ hexane $/{ }^{i-} \mathrm{PrOH}$ $=75 / 25, \lambda=208 \mathrm{~nm}, 1.0 \mathrm{~mL} / \mathrm{min}) ; \mathrm{t}_{\mathrm{R}(\text { major })}=12.1 \mathrm{~min}, \mathrm{t}_{\mathrm{R}(\operatorname{minor})}=16.0 \mathrm{~min}, 84 \%$ ee.

(S)-4-(2-Chlorophenyl)-5-nitropentan-2-one (4b). Colorless oil $(56 \%),[\alpha]_{\mathrm{D}}{ }^{20}=+28.7$ (c 1.6, $\left.\mathrm{CHCl}_{3}\right) .{ }^{1} \mathrm{H}$ NMR $\left(400 \mathrm{MHz}, \mathrm{CDCl}_{3}\right): \delta$ 7.42-7.21 (4H, m, Ar-H), 4.80-4.72 (2H, m, $\left.\mathrm{CH}_{2} \mathrm{NO}_{2}\right)$, 4.50-4.43 (1H, m, CHAr), 3.07 (1H, dd, J = 18.0, 7.8 Hz, $\left.\mathrm{CH}_{2} \mathrm{CO}\right), 2.97(1 \mathrm{H}, \mathrm{dd}, \mathrm{J}=18.0,6.0 \mathrm{~Hz}$, $\left.\mathrm{CH}_{2} \mathrm{CO}\right), 2.17\left(3 \mathrm{H}, \mathrm{s}, \mathrm{CH}_{3}\right) ;{ }^{13} \mathrm{C} \mathrm{NMR}\left(100 \mathrm{MHz}, \mathrm{CDCl}_{3}\right): \delta 205.3(\mathrm{C}=\mathrm{O}), 136.0\left(\mathrm{C}_{\text {quat }}\right), 133.7$ $\left(\mathrm{C}_{\text {quat }}\right), 130.4(\mathrm{CH}), 129.0(\mathrm{CH}), 128.4(\mathrm{CH}), 127.4(\mathrm{CH}), 77.4\left(\mathrm{CH}_{2}\right), 44.5\left(\mathrm{CH}_{2}\right), 35.8(\mathrm{CH})$, $30.2\left(\mathrm{CH}_{3}\right)$; IR (KBr): 2923, 2853, 1714, 1552, 1384, 1165, 1033, 753, $686 \mathrm{~cm}^{-1}$; MS (EI): 241.1 $\left(\mathrm{M}^{+}\right)$, 205.9, 160.0, 158.9, 144.9, 137.9, 114.9, 76.9; HRMS (ESI) calcd for $\mathrm{C}_{11} \mathrm{H}_{12} \mathrm{NO}_{3} \mathrm{Cl}$ $(\mathrm{M}+\mathrm{Na})^{+}: 264.0403$, found: 264.0401 . The enantiomeric excess was determined by HPLC with an AS-H column $\left({ }^{n-}\right.$ hexane $\left./{ }^{i-} \mathrm{PrOH}=75 / 25, \lambda=208 \mathrm{~nm}, 1.0 \mathrm{~mL} / \mathrm{min}\right) ; \mathrm{t}_{\mathrm{R}(\text { major })}=10.5 \mathrm{~min}, \mathrm{t}_{\mathrm{R}(\text { minor })}$ $=12.2 \mathrm{~min}, 73 \%$ ee. 
(S)-4-(3-Chlorophenyl)-5-nitropentan-2-one (4c). ${ }^{8 \mathrm{~b}}$ Colorless oil $(66 \%),[\alpha]_{\mathrm{D}}{ }^{20}=+1.2$ (c 2.0 , $\left.\mathrm{CHCl}_{3}\right) .{ }^{1} \mathrm{H}$ NMR $\left(400 \mathrm{MHz}, \mathrm{CDCl}_{3}\right): \delta$ 7.27-7.10 (4H, m, Ar-H), $4.69(1 \mathrm{H}, \mathrm{dd}, \mathrm{J}=12.6,6.6 \mathrm{~Hz}$, $\left.\mathrm{CH}_{2} \mathrm{NO}_{2}\right), 4.58\left(1 \mathrm{H}, \mathrm{dd}, \mathrm{J}=12.4,8.0 \mathrm{~Hz}, \mathrm{CH}_{2} \mathrm{NO}_{2}\right), 4.03-3.93(1 \mathrm{H}, \mathrm{m}, \mathrm{CHAr}), 2.90$ (2H, d, J = $\left.7.0 \mathrm{~Hz}, \mathrm{CH}_{2} \mathrm{CO}\right), 2.13\left(3 \mathrm{H}, \mathrm{s}, \mathrm{CH}_{3}\right) ;{ }^{13} \mathrm{C} \mathrm{NMR}\left(100 \mathrm{MHz}, \mathrm{CDCl}_{3}\right): \delta 204.9(\mathrm{C}=\mathrm{O}), 141.0\left(\mathrm{C}_{\text {quat }}\right)$, $134.8\left(\mathrm{C}_{\text {quat }}\right), 130.3(\mathrm{CH}), 128.1(\mathrm{CH}), 127.6(\mathrm{CH}), 125.8(\mathrm{CH}), 79.0\left(\mathrm{CH}_{2}\right), 45.9\left(\mathrm{CH}_{2}\right), 38.6$ (CH), $30.3\left(\mathrm{CH}_{3}\right)$; IR (KBr): 2923, 2853, 1734, 1559, 1384, 1160, 782, $695 \mathrm{~cm}^{-1}$; MS (EI): 240.9 $\left(\mathrm{M}^{+}\right), 195.9,193.9,159.0,137.9,114.9,76.9$; HRMS (ESI) calcd for $\mathrm{C}_{11} \mathrm{H}_{12} \mathrm{NO}_{3} \mathrm{Cl}(\mathrm{M}+\mathrm{Na})^{+}$: 264.0403, found: 264.0385; The enantiomeric excess was determined by HPLC with an AS-H column $\left({ }^{n}\right.$ hexane $\left./{ }^{i} \mathrm{PrOH}=75 / 25, \lambda=208 \mathrm{~nm}, 1.0 \mathrm{~mL} / \mathrm{min}\right) ; \mathrm{t}_{\mathrm{R}(\text { major })}=13.0 \mathrm{~min}, \mathrm{t}_{\mathrm{R}(\text { minor })}=19.8$ $\min , 66 \%$ ee.

(S)-4-(4-Chlorophenyl)-5-nitropentan-2-one (4d). ${ }^{8 \mathrm{~b}}$ White solid $(60 \%)$, m.p. $88-90{ }^{\circ} \mathrm{C},[\alpha]_{\mathrm{D}}{ }^{20}$ $=+2.0\left(\mathrm{c} 1.5, \mathrm{CHCl}_{3}\right) .{ }^{1} \mathrm{H} \mathrm{NMR}\left(400 \mathrm{MHz}, \mathrm{CDCl}_{3}\right): \delta 7.30(2 \mathrm{H}, \mathrm{d}, \mathrm{J}=8.2 \mathrm{~Hz}, \mathrm{Ar}-\mathrm{H}), 7.16(2 \mathrm{H}, \mathrm{d}$, $\mathrm{J}=8.2 \mathrm{~Hz}, \mathrm{Ar}-\mathrm{H}), 4.68\left(1 \mathrm{H}, \mathrm{dd}, \mathrm{J}=12.4,6.6 \mathrm{~Hz}, \mathrm{CH}_{2} \mathrm{NO}_{2}\right), 4.57(1 \mathrm{H}, \mathrm{dd}, \mathrm{J}=12.4,7.8 \mathrm{~Hz}$, $\left.\mathrm{CH}_{2} \mathrm{NO}_{2}\right), 4.00(1 \mathrm{H}, \mathrm{m}, \mathrm{CHAr}), 2.89\left(2 \mathrm{H}, \mathrm{d}, \mathrm{J}=7.0 \mathrm{~Hz}, \mathrm{CH}_{2} \mathrm{CO}\right), 2.13\left(3 \mathrm{H}, \mathrm{s}, \mathrm{CH}_{3}\right) ;{ }^{13} \mathrm{C}$ NMR $\left(100 \mathrm{MHz}, \mathrm{CDCl}_{3}\right): \delta 205.0(\mathrm{C}=\mathrm{O}), 137.3\left(\mathrm{C}_{\text {quat }}\right), 133.8\left(\mathrm{C}_{\text {quat }}\right), 129.3(2 \mathrm{CH}), 128.8(2 \mathrm{CH}), 79.2$ $\left(\mathrm{CH}_{2}\right), 46.0\left(\mathrm{CH}_{2}\right), 38.4(\mathrm{CH}), 30.4\left(\mathrm{CH}_{3}\right)$; IR $(\mathrm{KBr}): 3029,2948,2924,2853,1913,1717,1553$, 1491, 1384, 1362, 1166, 854, 818, 734, $715 \mathrm{~cm}^{-1}$; MS (EI) : 241.1 (M $\left.{ }^{+}\right), 193.9,178.9,137.9$, 114.9; The enantiomeric excess was determined by HPLC with an AS-H column $\left({ }^{n}{ }^{-}\right.$hexane $\left./{ }^{i} \mathrm{PrOH}=75 / 25, \lambda=208 \mathrm{~nm}, 1.0 \mathrm{~mL} / \mathrm{min}\right) ; \mathrm{t}_{\mathrm{R}(\text { major })}=12.8 \mathrm{~min}, \mathrm{t}_{\mathrm{R}(\text { minor })}=19.2 \mathrm{~min}, 70 \%$ ee.

(S)-5-Nitro-4-(2-nitrophenyl)pentan-2-one (4e). ${ }^{8 \mathrm{a}}$ Yellow oil $(38 \%),[\alpha]_{\mathrm{D}}{ }^{20}=-84.0$ (c 0.5 , $\left.\mathrm{CHCl}_{3}\right) .{ }^{1} \mathrm{H}$ NMR $\left(400 \mathrm{MHz}, \mathrm{CHCl}_{3}\right): \delta 7.89(1 \mathrm{H}, \mathrm{d}, \mathrm{J}=8.0 \mathrm{~Hz}, \mathrm{Ar}-\mathrm{H}), 7.61-7.57(1 \mathrm{H}, \mathrm{m}, \mathrm{Ar}-\mathrm{H})$, 7.47-7.37 (2H, m, Ar-H), 4.87-4.79 (2H, m, $\left.\mathrm{CH}_{2} \mathrm{NO}_{2}\right)$, 4.55-4.50 (1H, m, CHAr), 3.05 (2H, d, J = $\left.6.8 \mathrm{~Hz}, \mathrm{CH}_{2} \mathrm{CO}\right), 2.16\left(3 \mathrm{H}, \mathrm{s}, \mathrm{CH}_{3}\right) ;{ }^{13} \mathrm{C} \mathrm{NMR}\left(100 \mathrm{MHz}, \mathrm{CHCl}_{3}\right): \delta 204.8(\mathrm{C}=\mathrm{O}), 149.9\left(\mathrm{C}_{\text {quat }}\right)$, $133.5\left(\mathrm{C}_{\text {quat }}\right), 133.3(\mathrm{CH}), 128.7(\mathrm{CH}), 128.6(\mathrm{CH}), 125.2(\mathrm{CH}), 77.9\left(\mathrm{CH}_{2}\right), 45.3\left(\mathrm{CH}_{2}\right), 33.9$ (CH), $30.1\left(\mathrm{CH}_{3}\right)$; IR (KBr): 2924, 2853, 1716, 1559, 1473, 1384, $710 \mathrm{~cm}^{-1}$; MS (EI): $251.9\left(\mathrm{M}^{+}\right)$, 206.8, 162.9, 157.0, 145.9, 129.9, 90.0, 76.9; The enantiomeric excess was determined by HPLC with an AS-H column $\left({ }^{n}\right.$ hexane $\left./{ }^{i-} \mathrm{PrOH}=75 / 25, \lambda=208 \mathrm{~nm}, 1.0 \mathrm{~mL} / \mathrm{min}\right) ; \mathrm{t}_{\mathrm{R}(\text { major })}=21.1 \mathrm{~min}$, $\mathrm{t}_{\mathrm{R}(\text { minor })}=25.1 \mathrm{~min}, 75 \%$ ee.

(S)-4-(4-Methoxyphenyl)-5-nitropentan-2-one (4f). ${ }^{4 \mathrm{a}, 8 \mathrm{a}}$ White solid (58\%), m.p. $86-88{ }^{\circ} \mathrm{C}$ (lit. ${ }^{4 \mathrm{a}}$ m.p. 93.5-94.5 $\left.{ }^{\circ} \mathrm{C}\right),[\alpha]_{\mathrm{D}}{ }^{20}=-1.3\left(\right.$ c $\left.1.8, \mathrm{CHCl}_{3}\right)\left(\right.$ lit. $^{4 \mathrm{a}}[\alpha]_{\mathrm{D}}{ }^{20}=-1.5$, c $1.32, \mathrm{CHCl}_{3}, 99 \%$ ee); ${ }^{1} \mathrm{H}$ NMR (400 MHz, CDCl $)$ ): $\delta 7.14(2 \mathrm{H}, \mathrm{d}, \mathrm{J}=8.6 \mathrm{~Hz}, \mathrm{Ar}-\mathrm{H}), 6.86(2 \mathrm{H}, \mathrm{d}, \mathrm{J}=8.6 \mathrm{~Hz}, \mathrm{Ar}-\mathrm{H}), 4.66$ $\left(1 \mathrm{H}, \mathrm{dd}, \mathrm{J}=12.2,6.8 \mathrm{~Hz}, \mathrm{CH}_{2} \mathrm{NO}_{2}\right), 4.56\left(1 \mathrm{H}, \mathrm{dd}, \mathrm{J}=12.2,7.8 \mathrm{~Hz}, \mathrm{CH}_{2} \mathrm{NO}_{2}\right), 4.01(1 \mathrm{H}, \mathrm{m}$, $\mathrm{CHAr}), 3.78\left(3 \mathrm{H}, \mathrm{s}, \mathrm{OCH}_{3}\right), 2.89\left(2 \mathrm{H}, \mathrm{J}=7.0 \mathrm{~Hz}, \mathrm{CH}_{2} \mathrm{CO}\right), 2.10\left(3 \mathrm{H}, \mathrm{s}, \mathrm{CH}_{3}\right) ;{ }^{13} \mathrm{C} \mathrm{NMR}(100$ $\left.\mathrm{MHz}, \mathrm{CDCl}_{3}\right): \delta 205.5(\mathrm{C}=\mathrm{O}), 159.1\left(\mathrm{C}_{\text {quat }}\right), 130.6\left(\mathrm{C}_{\text {quat }}\right), 128.4(2 \mathrm{CH}), 114.4(2 \mathrm{CH}), 79.7\left(\mathrm{CH}_{2}\right)$, $55.3\left(\mathrm{CH}_{3}\right), 46.3\left(\mathrm{CH}_{2}\right), 38.4(\mathrm{CH}), 30.4\left(\mathrm{CH}_{3}\right)$; IR $(\mathrm{KBr}): 3003,2962,2838,1714,1550,1519$, 1383, 1262, 1179, 1034, 819, $730 \mathrm{~cm}^{-1}$; MS (EI): $237.0\left(\mathrm{M}^{+}\right), 191.0,190.0,174.9,148.0,134.0$, 90.9, 76.9; The enantiomeric excess was determined by HPLC with an AS-H column $\left({ }^{n-}\right.$ hexane $\left./{ }^{i-} \mathrm{PrOH}=75 / 25, \lambda=208 \mathrm{~nm}, 1.0 \mathrm{~mL} / \mathrm{min}\right) ; \mathrm{t}_{\mathrm{R}(\text { major })}=20.9 \mathrm{~min}, \mathrm{t}_{\mathrm{R}(\text { minor })}=45.8 \mathrm{~min}, 84 \%$ ee. 
(S)-4-(4-Methylphenyl)-5-nitropentan-2-one (4g). ${ }^{4 \mathrm{a}}$ White solid (58\%), m.p. $65-67{ }^{\circ} \mathrm{C},[\alpha]_{\mathrm{D}}{ }^{20}=$ $+1.3\left(\mathrm{c} 1.6, \mathrm{CHCl}_{3}\right)\left(\right.$ lit. $^{4 \mathrm{a}}[\alpha]_{\mathrm{D}}{ }^{20}=+5.5, \mathrm{c} 1.59, \mathrm{CHCl}_{3}, 97 \%$ ee $) .{ }^{1} \mathrm{H}$ NMR $\left(400 \mathrm{MHz}, \mathrm{CDCl}_{3}\right): \delta$ 7.26-7.08 (4H, m, Ar-H), $4.67\left(1 \mathrm{H}, \mathrm{dd}, \mathrm{J}=12.2,6.8 \mathrm{~Hz}, \mathrm{CH}_{2} \mathrm{NO}_{2}\right), 4.57(1 \mathrm{H}, \mathrm{dd}, \mathrm{J}=12.2,7.6 \mathrm{~Hz}$, $\left.\mathrm{CH}_{2} \mathrm{NO}_{2}\right), 4.00-3.92(1 \mathrm{H}, \mathrm{m}, \mathrm{CHAr}), 2.89\left(2 \mathrm{H}, \mathrm{d}, \mathrm{J}=7.0 \mathrm{~Hz}, \mathrm{CH}_{2} \mathrm{CO}\right), 2.31\left(3 \mathrm{H}, \mathrm{s}, \mathrm{ArCH}_{3}\right), 2.11$ $\left(3 \mathrm{H}, \mathrm{s}, \mathrm{CH}_{3}\right) ;{ }^{13} \mathrm{C} \mathrm{NMR}\left(100 \mathrm{MHz}, \mathrm{CDCl}_{3}\right): \delta 205.5(\mathrm{C}=\mathrm{O}), 137.6\left(\mathrm{C}_{\text {quat }}\right), 135.7\left(\mathrm{C}_{\text {quat }}\right), 129.7$ $(2 \mathrm{CH}), 127.2(2 \mathrm{CH}), 79.6\left(\mathrm{CH}_{2}\right), 46.2\left(\mathrm{CH}_{2}\right), 38.7(\mathrm{CH}), 30.4\left(\mathrm{CH}_{3}\right), 21.0\left(\mathrm{CH}_{3}\right)$; $\mathrm{IR}(\mathrm{KBr})$ : 3030, 3007, 2950, 2922, 1710, 1552, 1373, 1206, 805, $716 \mathrm{~cm}^{-1}$; MS (EI): $221.0\left(\mathrm{M}^{+}\right), 186.9,174.0$, 158.9, 117.0, 132.0, 90.9, 76.9. The enantiomeric excess was determined by HPLC with an AS-H column $\left({ }^{n}\right.$ hexane $\left.\left.{ }^{j-} \mathrm{PrOH}=75 / 25, \lambda=208 \mathrm{~nm}, 1.0 \mathrm{~mL} / \mathrm{min}\right) ; \mathrm{t}_{\mathrm{R}(\text { major })}=10.6 \mathrm{~min}, \mathrm{t}_{\mathrm{R}(\text { minor }}\right)=15.6$ $\min , 68 \%$ ee.

(R)-4-(Furan-2-yl)-5-nitropentan-2-one (4h). ${ }^{3 \mathrm{j}, 4 \mathrm{a}, 5 \mathrm{~b}, 8 \mathrm{a}}$ White solid (72\%), m.p. $49-51{ }^{\circ} \mathrm{C}$ (lit. ${ }^{4 a}$ m.p. $\left.54.5-56{ }^{\circ} \mathrm{C}\right),[\alpha]_{\mathrm{D}}{ }^{20}=+4.5\left(\mathrm{c} 1.5, \mathrm{CHCl}_{3}\right)\left(\mathrm{lit}^{4 \mathrm{a}}\right.$. $[\alpha]_{\mathrm{D}}{ }^{20}=+5.7$, c $1.32, \mathrm{CHCl}_{3}, 99 \%$ ee). ${ }^{1} \mathrm{H}$ NMR (400 MHz, $\left.\mathrm{CDCl}_{3}\right): \delta 7.34(1 \mathrm{H}, \mathrm{m}, \mathrm{ArH}), 6.30(1 \mathrm{H}, \mathrm{m}, \mathrm{Ar}-\mathrm{H}), 6.14(1 \mathrm{H}, \mathrm{m}, \mathrm{Ar}-\mathrm{H}), 4.70$ $\left(1 \mathrm{H}, \mathrm{dd}, \mathrm{J}=12.4,6.4 \mathrm{~Hz}, \mathrm{CH}_{2} \mathrm{NO}_{2}\right), 4.66\left(1 \mathrm{H}, \mathrm{dd}, \mathrm{J}=8.6,3.0 \mathrm{~Hz}, \mathrm{CH}_{2} \mathrm{NO}_{2}\right), 4.10(1 \mathrm{H}, \mathrm{m}$, CHAr), $2.94\left(2 \mathrm{H}, \mathrm{d}, \mathrm{J}=18.0,6.4 \mathrm{~Hz}, \mathrm{CH}_{2} \mathrm{CO}\right), 2.18\left(3 \mathrm{H}, \mathrm{s}, \mathrm{CH}_{3}\right) ;{ }^{13} \mathrm{C} \mathrm{NMR}\left(100 \mathrm{MHz}, \mathrm{CDCl}_{3}\right)$ : $\delta 205.1(\mathrm{C}=\mathrm{O}), 151.7\left(\mathrm{C}_{\text {quat }}\right), 142.3(\mathrm{CH}), 110.5(\mathrm{CH}), 107.1(\mathrm{CH}), 77.4\left(\mathrm{CH}_{2}\right), 43.5\left(\mathrm{CH}_{2}\right), 32.9$ (CH), $30.2\left(\mathrm{CH}_{3}\right)$; IR (KBr): 3123, 3006, 2920, 1718, 1554, 1378, 1164, 1015, $742 \mathrm{~cm}^{-1}$; MS (EI): $197.0\left(\mathrm{M}^{+}\right), 150.9,149.9,134.9,107.9,93.9,76.9$. The enantiomeric excess was determined by HPLC with an OD-H column $\left({ }^{n}\right.$ hexane $\left.{ }^{j-} \mathrm{PrOH}=94 / 6, \lambda=208 \mathrm{~nm}, 0.6 \mathrm{~mL} / \mathrm{min}\right) ; \mathrm{t}_{\mathrm{R}(\text { major })}=35.1$ $\min , \mathrm{t}_{\mathrm{R}(\operatorname{minor})}=37.2 \mathrm{~min}, 75 \%$ ee.

(R)-5-Nitro-4-(thiophen-2-yl)pentan-2-one (4i). . $^{3 \mathrm{j}, 4 \mathrm{a}, 5 \mathrm{a}-\mathrm{b}, 8 \mathrm{a}}$ Yellow oil (70\%), $[\alpha]_{\mathrm{D}}{ }^{20}=+6.1$ (c 1.8, $\left.\mathrm{CHCl}_{3}\right) ;{ }^{1} \mathrm{H}$ NMR $\left(400 \mathrm{MHz}, \mathrm{CDCl}_{3}\right): \delta 7.20(1 \mathrm{H}, \mathrm{d}, \mathrm{J}=5.0 \mathrm{~Hz}, \mathrm{Ar}-\mathrm{H}), 6.94-6.90(2 \mathrm{H}, \mathrm{m}, \mathrm{Ar}-\mathrm{H})$, $4.72\left(1 \mathrm{H}, \mathrm{dd}, \mathrm{J}=12.4,6.4 \mathrm{~Hz}, \mathrm{CH}_{2} \mathrm{NO}_{2}\right), 4.61\left(1 \mathrm{H}, \mathrm{dd}, \mathrm{J}=12.4,7.4 \mathrm{~Hz}, \mathrm{CH}_{2} \mathrm{NO}_{2}\right), 4.32(1 \mathrm{H}, \mathrm{m}$, CHAr), $2.97\left(2 \mathrm{H}, \mathrm{d}, \mathrm{J}=6.8 \mathrm{~Hz}, \mathrm{CH}_{2} \mathrm{CO}\right), 2.16\left(3 \mathrm{H}, \mathrm{s}, \mathrm{CH}_{3}\right) ;{ }^{13} \mathrm{C}$ NMR $\left(100 \mathrm{MHz}, \mathrm{CDCl}_{3}\right): \delta$ $205.0(\mathrm{C}=\mathrm{O}), 141.6\left(\mathrm{C}_{\text {quat }}\right), 127.2(\mathrm{CH}), 125.5(\mathrm{CH}), 124.7(\mathrm{CH}), 79.7\left(\mathrm{CH}_{2}\right), 46.8\left(\mathrm{CH}_{2}\right), 34.4$ (CH), $30.3\left(\mathrm{CH}_{3}\right)$; IR (KBr): 3108, 3006, 2920, 1715, 1551, 1431, 1380, 1166, 851, $706 \mathrm{~cm}^{-1}$; MS (EI): $213.0\left(\mathrm{M}^{+}\right), 166.9$. 165.9, 150.9, 123.9, 109.9, 83.9. The enantiomeric excess was determined by HPLC with an AS-H column $\left({ }^{n}\right.$ hexane $\left./{ }^{i} \mathrm{PrOH}=75 / 25, \lambda=208 \mathrm{~nm}, 1.0 \mathrm{~mL} / \mathrm{min}\right)$; $\mathrm{t}_{\mathrm{R}(\text { major })}=14.9$ min, $\mathrm{t}_{\mathrm{R}(\text { minor })}=19.7 \mathrm{~min}, 69 \%$ ee.

5-Nitro-4-phenylpentan-1-methoxyl-2-one $(\mathbf{4 j}){ }^{4 \mathrm{a}}$ White solid $(58 \%),[\alpha]_{\mathrm{D}}{ }^{20}=+1.2(\mathrm{c} 0.7$, $\mathrm{CHCl}_{3}$ ). IR (KBr): 2930, 2830, 1715, 1556, 1382, 1109, 763, $703 \mathrm{~cm}^{-1}$; MS (EI): $237.0\left(\mathrm{M}^{+}\right)$, 193.9, 146.9, 116.9, 103.9, 90.9, 76.9.

syn-isomer. ${ }^{1} \mathrm{H}$ NMR $\left(400 \mathrm{MHz}, \mathrm{CDCl}_{3}\right): \delta$ 7.35-7.28 (3H, m, Ar-H), 7.25-7.21 (2H, m, Ar-H), $4.93\left(1 \mathrm{H}, \mathrm{dd}, \mathrm{J}=12.8,8.2 \mathrm{~Hz}, \mathrm{CH}_{2} \mathrm{NO}_{2}\right), 4.65\left(1 \mathrm{H}, \mathrm{dd}, \mathrm{J}=12.8,6.8 \mathrm{~Hz}, \mathrm{CH}_{2} \mathrm{NO}_{2}\right), 3.93-3.80$ $\left(2 \mathrm{H}, \mathrm{m}, \mathrm{CH}_{2} \mathrm{CO}\right), 3.45\left(3 \mathrm{H}, \mathrm{s}, \mathrm{OCH}_{3}\right), 1.78\left(3 \mathrm{H}, \mathrm{s}, \mathrm{CH}_{3}\right) ;{ }^{13} \mathrm{C} \mathrm{NMR}\left(100 \mathrm{MHz}, \mathrm{CDCl}_{3}\right): \delta 209.9$ $(\mathrm{C}=\mathrm{O}), 134.5\left(\mathrm{C}_{\text {quat }}\right), 129.0(2 \mathrm{CH}), 128.5(2 \mathrm{CH}), 128.4(\mathrm{CH}), 86.4(\mathrm{CH}), 76.5\left(\mathrm{CH}_{2}\right), 59.8\left(\mathrm{CH}_{3}\right)$, $46.3(\mathrm{CH}), 26.4\left(\mathrm{CH}_{3}\right)$. The enantiomeric excess was determined by HPLC with an OD-H column $\left({ }^{n}{ }^{-}\right.$hexane $\left./{ }^{i-} \mathrm{PrOH}=75 / 25, \lambda=208 \mathrm{~nm}, 0.6 \mathrm{~mL} / \mathrm{min}\right) ; \mathrm{t}_{\mathrm{R}(\text { minor })}=13.8 \mathrm{~min}, \mathrm{t}_{\mathrm{R}(\text { major })}=26.3$ $\min , 75 \%$ ee.

anti-isomer. ${ }^{1} \mathrm{H}$ NMR $\left(400 \mathrm{MHz}, \mathrm{CDCl}_{3}\right): \delta$ 7.35-7.28 (3H, m, Ar-H), 7.25-7.21 (2H, m, Ar-H), 
$4.86\left(1 \mathrm{H}, \mathrm{dd}, \mathrm{J}=13.2,5.6 \mathrm{~Hz}, \mathrm{CH}_{2} \mathrm{NO}_{2}\right), 4.72\left(1 \mathrm{H}, \mathrm{dd}, \mathrm{J}=13.2,8.6 \mathrm{~Hz}, \mathrm{CH}_{2} \mathrm{NO}_{2}\right), 3.93-3.80$ $\left(2 \mathrm{H}, \mathrm{m}, \mathrm{CH}_{2} \mathrm{CO}\right), 3.38\left(3 \mathrm{H}, \mathrm{s}, \mathrm{OCH}_{3}\right), 2.04\left(3 \mathrm{H}, \mathrm{s}, \mathrm{CH}_{3}\right) ;{ }^{13} \mathrm{C} \mathrm{NMR}\left(100 \mathrm{MHz}, \mathrm{CDCl}_{3}\right): \delta 208.1$ $(\mathrm{C}=\mathrm{O}), 135.4\left(\mathrm{C}_{\text {quat }}\right), 129.1(2 \mathrm{CH}), 128.9(2 \mathrm{CH}), 128.1(\mathrm{CH}), 88.2(\mathrm{CH}), 76.5\left(\mathrm{CH}_{2}\right), 59.8\left(\mathrm{CH}_{3}\right)$, $45.9(\mathrm{CH}), 26.2\left(\mathrm{CH}_{3}\right)$. The enantiomeric excess was determined by HPLC with an OD-H column $\left({ }^{n}\right.$ hexane $\left.{ }^{j-} \mathrm{PrOH}=75 / 25, \lambda=208 \mathrm{~nm}, 0.6 \mathrm{~mL} / \mathrm{min}\right) ; \mathrm{t}_{\mathrm{R}(\text { minor })}=14.9 \mathrm{~min}, \mathrm{t}_{\mathrm{R} \text { (major) }}=19.6$ $\min , 26 \%$ ee.

2-(1-phenyl-2-nitroethyl)-cyclopentanone (4k). ${ }^{8 \mathrm{~m}-\mathrm{n}}$ White solid $(35 \%),[\alpha]_{\mathrm{D}}{ }^{20}=-3.3$ (c 1.3 , $\mathrm{CHCl}_{3}$ ); IR (KBr): 3063, 3031, 2983, 2923, 2080, 1730, 1552, 1159, 1131, 785, $697 \mathrm{~cm}^{-1}$; MS (EI): $233.1\left(\mathrm{M}^{+}\right), 186.1,157.9,129.0,114.9,104.0,90.9,76.9$.

syn-isomer. ${ }^{1} \mathrm{H}$ NMR $\left(400 \mathrm{MHz}, \mathrm{CDCl}_{3}\right)$ : $\delta$ 7.34-7.30 (3H, m, Ar-H), 7.19-7.16 (2H, m, Ar-H), 5.02-5.00 (2H, m, $\left.\mathrm{CH}_{2} \mathrm{NO}_{2}\right), 3.86-3.81(1 \mathrm{H}, \mathrm{m}, \mathrm{CHAr}), 2.55-2.49(1 \mathrm{H}, \mathrm{m}, \mathrm{CHCO}), 2.34-1.43$ $\left(6 \mathrm{H}, \mathrm{m}, 3 \mathrm{CH}_{2}\right) ;{ }^{13} \mathrm{C}$ NMR $\left(100 \mathrm{MHz}, \mathrm{CDCl}_{3}\right): \delta 219.1(\mathrm{C}=\mathrm{O}), 137.4\left(\mathrm{C}_{\text {quat }}\right), 129.0(2 \mathrm{CH}), 128.5$ $(2 \mathrm{CH}), 128.0(\mathrm{CH}), 78.3\left(\mathrm{CH}_{2}\right), 51.4(\mathrm{CH}), 44.0\left(\mathrm{CH}_{2}\right), 39.3\left(\mathrm{CH}_{2}\right), 27.0(\mathrm{CH}), 20.6\left(\mathrm{CH}_{2}\right)$. The enantiomeric excess was determined by HPLC with an OD-H column $\left({ }^{n-}\right.$ hexane $/{ }^{i-} \mathrm{PrOH}=75 / 25$, $\lambda=208 \mathrm{~nm}, 0.6 \mathrm{~mL} / \mathrm{min}) ; \mathrm{t}_{\mathrm{R}(\text { minor })}=11.3 \mathrm{~min}, \mathrm{t}_{\mathrm{R}(\text { major })}=18.4 \mathrm{~min}, 84 \%$ ee.

anti-isomer. ${ }^{1} \mathrm{H}$ NMR $\left(400 \mathrm{MHz}, \mathrm{CDCl}_{3}\right): \delta$ 7.34-7.30 (3H, m, Ar-H), 7.19-7.16 $(2 \mathrm{H}, \mathrm{m}, \mathrm{Ar}-\mathrm{H})$, $5.33\left(1 \mathrm{H}, \mathrm{dd}, \mathrm{J}=12.8,5.6 \mathrm{~Hz}, \mathrm{CH}_{2} \mathrm{NO}_{2}\right), 4.71\left(1 \mathrm{H}, \mathrm{dd}, \mathrm{J}=12.8,10.0 \mathrm{~Hz}, \mathrm{CH}_{2} \mathrm{NO}_{2}\right), 3.73-3.67$ $(1 \mathrm{H}, \mathrm{m}, \mathrm{CHAr}), 2.43-2.36(1 \mathrm{H}, \mathrm{m}, \mathrm{CHCO}), 2.34-1.43\left(6 \mathrm{H}, \mathrm{m}, 3 \mathrm{CH}_{2}\right) ;{ }^{13} \mathrm{C}$ NMR $(100 \mathrm{MHz}$, $\left.\mathrm{CDCl}_{3}\right): \delta 218.5(\mathrm{C}=\mathrm{O}), 137.7\left(\mathrm{C}_{\text {quat }}\right), 128.9(2 \mathrm{CH}), 128.0(2 \mathrm{CH}), 127.9(\mathrm{CH}), 78.3\left(\mathrm{CH}_{2}\right), 50.5$ $(\mathrm{CH}), 44.2\left(\mathrm{CH}_{2}\right), 38.7\left(\mathrm{CH}_{2}\right), 28.3(\mathrm{CH}), 20.3\left(\mathrm{CH}_{2}\right)$. The enantiomeric excess was determined by HPLC with an OD-H column $\left({ }^{n}\right.$ hexane $\left.{ }^{i-} \mathrm{PrOH}=75 / 25, \lambda=208 \mathrm{~nm}, 0.6 \mathrm{~mL} / \mathrm{min}\right) ; \mathrm{t}_{\mathrm{R}(\text { minor })}=$ 12.9 min, $\mathrm{t}_{\mathrm{R}(\text { major })}=14.4 \mathrm{~min}, 74 \%$ ee.

\section{Acknowledgements}

Financial support of this study from the National Natural Science Foundation of China (No. 20772160), the Ministry of Education (NCET project) and the Zhuhai Bureau of Science and Technology is gratefully acknowledged.

\section{References and Notes}

1. For the general reviews of organocatalysis, see: (a) Guillena, G.; Najera, C.; Ramon, D. J. Tetrahedron: Asymmetry 2007, 18, 2249. (b) Dalko, P. I. Enantioselective Organocatalysis; Wiley-VCH, Weinheim, 2007. (c) Yu, X. H.; Wang, W. Chem. Asian J. 2008, 3, 516.

2. For the reviews of chiral bifunctional organocatalysts, see: (a) Doyle, A. G.; Jacobsen, E. 
N. Chem. Rev. 2007, 107, 5713. (b) Connon, S. J. Chem. Eur. J. 2006, 12, 5418.

3. For the selected applications of chiral bifunctional catalysts, see: (a) Fang, Y. Q.; Jacobsen, E. N. J. Am. Chem. Soc. 2008, 130, 5660. (b) Wang, C. J.; Dong, X. Q.; Zhang, Z. H.; Xue, Z. Y.; Teng, H. L. J. Am. Chem. Soc. 2008, 130, 8606. (c) Rabalakos, C.; Wulff, W. D. J. Am. Chem. Soc. 2008, 130, 13524. (d) Wang, F.; Xiong, Y.; Liu, X. H; Feng, X. M. Adv. Synth. Catal. 2007, 349, 2665. (e) Sibi, M. P.; Itoh, K. J. Am. Chem. Soc. 2007, 129, 8064. (f) Pan, S. C.; Zhou, J.; List, B. Angew. Chem. Int. Ed. 2007, 46, 612. (g) Liu, K.; Cui, H. F.; Nie, J.; Dong, K. Y.; Li, X. J.; Ma, J. A. Org. Lett. 2007, 9, 923. (h) Tillman, A. L.; Ye, J. X.; Dixon, D. J. Chem. Commun. 2006, 1191. (i) Dixon, D. J.; Richardson, R. D. Synlett 2006, 81.

4. Jacobsen's bifunctional catalysts for the addition of aldehydes and ketones to nitroalkenes, see: (a) Huang, H. B.; Jacobsen, E. N. J. Am. Chem. Soc. 2006, 128, 7170. (b) Lalonde, M. P.; Chen, Y. G.; Jacobsen, E. N. Angew. Chem. Int. Ed. 2006, 45, 6366.

5. (a) Wei, S. W.; Yalalov, D. A.; Tsogoeva, S. B. Catalysis Today 2007, 121, 151. (b) Tsogoeva, S. B.; Wei, S. W. Chem. Commun. 2006, 1451. (c) Yalalov, D. A.; Tsogoeva, S. B.; Schmatz S. Adv. Synth. Catal. 2006, 348, 826.

6. Jiang, X. X.; Zhang, Y. F.; Chan, Albert S. C.; Wang, R. Org. Lett. 2009, 11, 153.

7. For recent reviews of organocatalytic asymmetric conjugate addition, see: (a) Almasi D.; Alonso D. A.; Najera C. Tetrahedron: Asymmetry 2007, 18, 299. (b) Tsogoeva S. B. Eur. J. Org. Chem. 2007, 11, 1701. (c) Dalko P. I.; Moisan L. Angew. Chem. Int. Ed. 2004, 43, 5138.

8. For selected references for the asymmetric conjugate addition of aldehydes and ketones to nitroalkenes using other organocatalysts, see: (a) Xue, F.; Zhang, S. L.; Duan, W. H.; Wang, W. Adv. Synth. Catal. 2008, 350, 2194. (b) Mandal, T.; Zhao, C. C. Angew. Chem. Int. Ed. 2008, 47, 7714. (c) Wiesner, M.; Revell, J. D.; Wennemers, H. Angew. Chem. Int. Ed. 2008, 47, 1871. (d) García-García, P.; Ladépêche, A.; Halder, R.; List, B. Angew. Chem. Int. Ed. 2008, 47, 4719. (e) Hayashi, Y.; Itoh,T.; Ohkubo, M.; Ishikawa, H. Angew. Chem. Int. Ed. 2008, 47, 4722. (f) Alcaide, B.; Almendros, P. Angew. Chem. Int. Ed. 2008, 47, 4632. (g) Chi, Y.; Guo, L.; Kopf, N. A.; Gellman, S. H. J. Am. Chem. Soc. 2008, 130, 5608. (h) Tan, B.; Chua, P. J.; Li, Y. X.; Zhong, G. F. Org. Lett. 2008, 10, 2437. (i) Belot, S.; Sulzer-Mosse, S.; Kehrli, S.; Alexakis, A. Chem. Commun. 2008, 4694. (j) Xiong, Y.; Wen, Y. H.; Wang, F.; Gao, B.; Liu, X. H.; Huang, X.; Feng, X. M. Adv. Synth. Catal. 2007, 349, 2156. (k) Ramasastry, S. S. V.; Zhang, H.; Tanaka, F.; Barbas III, C. F. J. Am. Chem. Soc. 2007, 129, 288. (1) Li, H.; Zu, L. S.; Xie, H. X.; Wang, J.; Jiang, W.; Wang, W. Org. Lett. 2007, 9, 1833. (m) Mase, N.; Watanabe, K.; Yoda, H.; Takabe, K.; Tanaka, F.; Barbas III, C. F. J. Am. Chem. Soc. 2006, 128, 4966. (n) Cao, C. L.; Ye, M. C.; Sun, X. L.; Tang, Y. Org. Lett. 2006, 8, 2901.

9. Zhang, X. J.; Liu, S. P.; Li, X. M.; Yan, M.; Chan, A. S. C. Chem. Commun. 2009, 833.

10. For the additives in the organocatalytic conjugate addition of aldehydes and ketones to nitroalkenes, see: (a) Chen, H. B.; Wang, Y.; Wei, S.Y.; Sun, J. Tetrahedron: Asymmetry 
2007, 18, 1308. (b) Vishnumaya; Singh, V. K. Org. Lett. 2007, 9, 1117. (c) Zhu, M. K.; Cun, L. F.; Mi, A. Q.; Jiang, Y. Z.; Gong, L. Z. Tetrahedron: Asymmetry 2006, 17, 491. (d) Andrey, O.; Alexakis, A.; Tomassini, A.; Bernardinelli, G. Adv. Synth. Catal. 2004, 346, 1147. (e) Alexakis, A.; Andrey, O. Org. Lett. 2002, 4, 3611. 